\title{
Prácticas de Lectura de Profesionales de la Comunicación en Uruguay: Áreas, Géneros y Estrategias
}

\author{
Damián DÍAZ* \\ Leticia LORIER** \\ Mariana ACHUGAR***
}

\begin{abstract}
* Profesor Asistente en la Facultad de Información y Comunicación (FIC), UdelaR. Es Magister en Lingüística Aplicada por la Universidad de Brasilia (2019) y Profesor de Portugués como Lengua Extranjera por el Instituto de Profesores Artigas (2014). Contacto: damian.diaz@fic.edu.uy.

** Profesora Agregada en la Facultad de Información y Comunicación (FIC), UdelaR. Es Licenciada en Comunicación por la Universidad de la República (2003). En la misma universidad posee estudios de maestría en lenguaje y cultura, posgrado en traducción literaria (2007), y TIC y educación superior (2009). Contacto: leticia.lorier@fic.edu.uy.
\end{abstract}

*** Profesora Agregada en la Facultad de Información y Comunicación (FIC), UdelaR. Es Doctora en Lingüística Hispánica por la Universidad de California (2002), Magister en Artes y Enseñanza por la School for International Training (1995) y Licenciada en lingüística por la Universidad de la República (1987). Contacto: mariana.achugar@fic.edu.uy.

\section{Resumen:}

Este artículo presenta los resultados generales de una encuesta sobre lectura realizada a profesionales en actuación, egresados de la licenciatura en Comunicación de una universidad pública de Uruguay. La encuesta forma parte de una investigación-acción iniciada en 2020 sobre literacidad disciplinar (HYLAND, 2004; MOJE, 2007; ACHUGAR; STAINTON, 2010; ACHUGAR; CARPENTER, 2012). El objetivo es describir las prácticas lectoras de profesionales del área (LEINHARDT; YOUNG, 1996; WINEBURG, 2001) y los géneros asociados (BAZERMAN, 2005; 2008; BAJTÍN, 2008; MARTIN; ROSE, 2008), para informar la planificación de los cursos de comprensión lectora de la misma facultad. El cuestionario de ítems deductivos e inductivos fue realizado por 82 profesionales, y sus resultados muestran que la lectura en el contexto profesional se realiza en varias lenguas, en géneros periodísticos, académicos y profesionales, que son leídos para producir textos escritos, pero también orales. Sus principales objetivos incluyen encontrar información general sobre hechos y su contexto y acceder a información teórica. Las prácticas y estrategias más utilizadas son contextualizar históricamente los textos, extraer su orientación, analizar su material gráfico y la confiabilidad de sus fuentes, analizar su prestigio institucional, el de los autores citados, la calidad de sus evidencias y la de sus argumentaciones.

\section{Palabras clave:}

Prácticas lectoras. Actividad profesional de la comunicación. Literacidad disciplinar.

Signum: Estudos da Linguagem, Londrina, v.24, n.1, p.162-181, abr. 2021 


\section{Prácticas de Lectura de Profesionales de la Comunicación en Uruguay: Áreas, Géneros y Estrategias}

Damián Díaz; Leticia Lorier; Mariana Achugar

\section{INTRODUCCIÓN}

Aprender una disciplina implica, no solo incorporar ciertos conocimientos, sino también nuevas formas de usar el lenguaje, que responden a los objetivos y características de una comunidad de práctica profesional. La literacidad disciplinar integra el conocimiento especializado de contenidos, de vocabulario técnico, de usos gramaticales particulares y de formas de razonamiento utilizadas para construir conocimiento en la disciplina (HYLAND, 2004). Es decir, estas prácticas letradas están asociadas a actividades sociales que permiten alcanzar objetivos en un contexto cultural determinado. Las prácticas letradas, entonces, son sensibles a los cambios socio-históricos y culturales por lo que es necesario explorar de manera situada estas formas de ser y hacer en las disciplinas. Tener acceso a esas prácticas disciplinares permite a los novatos el uso y el cuestionamiento crítico del conocimiento y de las prácticas de producción del mismo. Desde esta perspectiva socio-semiótica y socio-cultural de la literacidad disciplinar (ACHUGAR; STAINTON, 2010; ACHUGAR; CARPENTER, 2012), enseñar a leer busca visibilizar y democratizar el acceso a las herramientas y las prácticas de producción de conocimiento (MOJE, 2007).

Este artículo presenta el análisis general de una encuesta a profesionales egresados de la licenciatura en Comunicación de una universidad pública de Uruguay, que actúan en las diferentes áreas en que este campo se divide (comunicación organizacional, periodismo, audiovisual, comunicación educativa y comunitaria, tic y multimedia, gestión cultural, investigación y docencia). La investigación integra un proyecto denominado Abordaje integral de la lectura: una innovación curricular y didáctica en cursos de lenguas en un contexto de numerosidad y heterogeneidad, iniciado en 2020. Este proyecto busca incorporar una perspectiva de literacidad disciplinar a la enseñanza de la comprensión lectora (español, inglés y portugués) en las carreras de grado de nuestra facultad. El objetivo de la encuesta sobre la que informamos en este artículo es obtener un panorama preliminar de las prácticas de lectura en primera lengua (L1) y en lenguas adicionales (L2) de los profesionales de la comunicación egresados de la FIC, que, junto con subsiguientes entrevistas a informantes calificados y observaciones de prácticas en el contexto universitario, nos permitan orientar la planificación de los cursos de lectura.

Consideramos que la conciencia metacognitiva de los encuestados brinda insumos para identificar prácticas de lectura asociadas con las esferas de profesionales de la comunicación en Uruguay en este momento histórico. Asimismo, conocer los sistemas de actividades, los géneros y sus sistemas de organización muestra en parte lo que constituye la práctica letrada en esta colectividad profesional (BAZERMAN, 2005; 2008). Mapear las prácticas lectoras utilizadas en esta comunidad nos permitirá seleccionar géneros, estrategias y disposiciones que acerquen las prácticas de aula a la praxis profesional ${ }^{1}$, así como guiarnos en el trazado de perfiles de actor "experto” y en la definición de criterios de evaluación.

\footnotetext{
${ }^{1}$ Definimos prácticas lectoras como las formas de abordar la lectura en el contexto de una comunidad interpretativa. Ellas incluyen objetivos particulares y formas de aproximarse a los textos para interpretarlos y evaluarlos. Estrategias de lectura refiere a planes y herramientas metacognitivas utilizados para facilitar el procesamiento de la información y la comprensión. Las disposiciones refieren a las orientaciones de los lectores hacia el texto, que pueden ser críticas, pasivas o convergentes con interpretaciones y significados del texto.
} 
Esto es de especial relevancia ya que los docentes de los cursos de lectura no somos, necesariamente, profesionales de la comunicación, y nuestras prácticas letradas no coinciden con las del área de formación de los alumnos (LABELLA-SÁNCHEZ; CRISTOVÃO, 2015). Además, aunque existen algunos trabajos que han estudiado la literacidad en la formación para el periodismo (DI CAPUA-HIDALGO, 2016a), no existen investigaciones relacionadas con las prácticas letradas de los comunicadores en su actividad fuera del ámbito académico ${ }^{2}$, accionar que constituye un objeto de estudio complejo por su naturaleza multifacética y transdisciplinaria (FRANCO, 2019) y porque los profesionales de la comunicación realizan sus actividades en áreas muy diversas. Tampoco existen muchos estudios previos en Latinoamérica enfocados en la lectura disciplinar (NAVARRO et al., 2016). En ese sentido, este trabajo contribuye a la elaboración preliminar de un constructo de literacidad disciplinar para el área, que sea útil no sólo a nuestros cursos de lectura, sino a la comprensión del métier de los comunicadores.

En ese marco, nos preguntamos: 1) ¿Cuáles son las prácticas lectoras de los profesionales de la comunicación?, y 2) ¿Qué géneros y estrategias de comprensión lectora se asocian con esas prácticas?

Organizamos este artículo en 5 secciones: una introducción; una breve exposición teórica; una exposición de los aspectos metodológicos; un análisis de los resultados; y consideraciones finales y sus implicaciones didácticas.

\section{Prácticas Profesionales, Géneros y Literacidad}

Desde una perspectiva crítica y de justicia social, enseñar la literacidad disciplinar implica no solo enseñar herramientas lingüísticas y prácticas letradas asociadas con esferas con poder social como las académicas o las profesionales (MARTIN; ROSE, 2008), sino dar acceso a formas de construir conocimiento (MOJE, 2007). La búsqueda de un carácter democrático e igualitario de la educación supone un intento de aproximación a la cohesión social, es decir, a la posibilidad de que todas las personas puedan insertarse y participar de forma eficaz en las múltiples actividades colectivas que se desarrollan en nuestra sociedad (BRONCKART; DOLZ, 2002). En nuestro caso, es menester conocer las prácticas profesionales del área de la comunicación, como insumo para planificar la formación superior en lectura (español, inglés, portugués), así como generar oportunidades para reflexionar críticamente sobre ellas y ofrecer instancias para usarlas en un contexto que tenga incidencia en el mundo.

Relevar las prácticas letradas disciplinares permite identificar los géneros utilizados en contextos profesionales y las prácticas llevadas a cabo, para tomarlos como referencia para la transposición didáctica (DE PIETRO; SCHNEUWLY, 2019). De acuerdo con Bronckart y Dolz (2002), se debe comenzar por analizar las propiedades de las tareas o de las actividades colectivas para construir los objetos de enseñanza. El mismo abordaje sustenta el programa de investigación de psicólogos de la educación como Gaea Linehardt (LEINHARDT; STAINTON; VIRJIJ, 1994; LEINHARDT; YOUNG, 1996) y Sam Wineburg (WINEBURG, 1998, 2001; WINEBURG; REISMAN, 2015; REISMAN, 2012), que han estudiado la lectura y la escritura disciplinar de expertos y novatos. Desde la lingüística educacional, se han descrito los géneros académicos asociados con distintas áreas de conocimiento en investigaciones empíricas en contextos educativos y profesionales (HALLIDAY; MARTIN, 1993; MARTIN; ROSE, 2008; SCHLEPPEGRELL, 2004; 2011).

\footnotetext{
${ }^{2}$ Los únicos estudios con los que hemos tenido contacto sobre literacidad profesional del área de la comunicación son los de la denominada literacidad mediática (media literacy), que no derivan del análisis empírico de las formas de trabajo de estos profesionales, pues se centran en el procesamiento de los textos que emanan del sistema de medios por parte de los ciudadanos comunes (POTTER, 2010).
} 
En América Latina, Navarro et al. (2016) sintetizaron las investigaciones y proyectos de la región en los últimos 20 años y encontraron un creciente número de estudios de la lectura y de la escritura disciplinar en la educación superior que abordan estas prácticas como procesos situados, y que identifican características discursivas de estos espacios disciplinares. Su estudio muestra que se investigan y se diseñan programas educativos en base a géneros y a prácticas letradas identificadas en el estudio empírico de comunidades intelectuales o contextos académicos particulares. Se destacan los estudios, desde la lingüística, de la Gramática Sistémica Funcional, como los de Manghi (2013), Moyano (2000), Moss (2010). Desde una perspectiva más cuantitativa, se han realizado aportes empíricos a la descripción de la literacidad disciplinar en estudios de corpus. El trabajo de Parodi (2010) constituye un antecedente importante en la caracterización de los géneros discursivos disciplinares en español con un abordaje de lingüística computacional.

Estos estudios previos se asientan en las propuestas de Bajtín (2008) sobre la relación intrínseca entre las esferas de la actividad humana y las formas de organización del lenguaje. Para Bajtín, "Las diversas esferas de la actividad humana están todas relacionadas con el uso de la lengua. Por eso está claro que el carácter y las formas de este uso son tan variados como aquellas esferas [...]" (BAJTÍN, 2008, p. 245). De acuerdo con Bazerman (2008), las prácticas de lenguaje atraviesan tanto las formas de pensar como de actuar de los individuos y generan hechos sociales con consecuencias materiales. En ese marco, la diversificación y especificación de las actividades sociales especializa las prácticas habituales de producción de textos, que dan lugar a géneros, acciones y actividades estabilizadas. De acuerdo con Bazerman (2005), los individuos realizan sus acciones "a través de formas textuales estandarizadas, típicas y, por tanto, inteligibles, o géneros, que están relacionadas a otros textos y géneros que ocurren en circunstancias relacionadas. Juntos, los varios tipos de textos se acomodan en conjuntos de géneros dentro de un sistema de géneros, los cuales forman parte de los sistemas de actividades bumanas" (BAZERMAN, 2005, p. 22, grifos del autor, traducción de los autores).

Las prácticas profesionales ocurren en el marco de sistemas de actividades en que diversas acciones individuales realizan finalidades sociales por medio de textos. Ellos pertenecen a conjuntos de géneros que cada persona, ejerciendo un determinado papel social o profesional, utiliza para su trabajo, formando sistemas que definen sus relaciones de producción, circulación, secuenciación y complementación (BAZERMAN, 2005). Para Bazerman (2005, 2008), conocer las habilidades necesarias para utilizar los textos propios de una actividad profesional, así como las motivaciones, las situaciones, los participantes y las acciones asociados a ellos, equivale a conocer gran parte de lo que un individuo debe aprender para desempeñarse en ella (comprender, tener voz e incidir). Esta necesidad es mayor cuando esas prácticas son mediadas por la escritura, en que es mayor el peso de las formas estabilizadas y codificadas de acción por medio del lenguaje (BAZERMAN, 2008).

Las configuraciones de prácticas que caracterizan las disciplinas incluyen formas de comunicación, conocimientos y acuerdos sobre cómo negociar diferencias y argumentar posturas (HYLAND, 2015). Las disciplinas constituyen comunidades interpretativas (FISH, 1980) que comparten formas de interpretar textos mediante lazos intertextuales y encuadres, y construyen identidades disciplinares e individuales. El conocimiento compartido y las expectativas sobre cómo participar en formas culturalmente legitimadas se expresa a través de estas prácticas indexadas a identidades sociales y áreas profesionales.

Los estudios de literacidad disciplinar (disciplinary literacy) confirman la relación estrecha entre lenguaje y el rol profesional. No existe un discurso académico general, sino variedades de literacidad de acuerdo con la disciplina (HYLAND, 2002; 2004). La noción de especificidad permite reconocer la diversidad de formas de construir significados en las comunidades académicas por las que transitan estudiantes y profesionales en un mundo que varía también dentro de las disciplinas de acuerdo con las 
diferentes orientaciones y posicionamientos teóricos. Es decir, en contextos interdisciplinarios como la profesión de la comunicación se usa el lenguaje de diversas maneras.

Hyland $(2008$, 2015) describe el discurso disciplinar como una práctica aproximada de convenciones retóricas que permiten posicionarse sobre temas relevantes y valorados mediante formas reconocidas de construir significados. De acuerdo con Shanahan y Shanahan (2012, p. 9, traducción de los autores), "las disciplinas difieren ostensiblemente en sus propósitos fundamentales, en sus géneros especializados, en sus artefactos simbólicos, en sus tradiciones de comunicación, en sus evaluaciones de estándares de calidad y precisión, y en su uso del lenguaje”. Por ello, la enseñanza de la lectura o de la escritura debe tener en cuenta el "saber hacer" de los expertos en cada área. De acuerdo con Shanahan y Shanahan (2012, p. 10, traducción de los autores), “a medida que los estudiantes examinan elecciones disciplinarias variadas o patrones relativamente especializados de uso del lenguaje, se pueden tornar mejor instrumentalizados para lidiar con las demandas de las disciplinas particulares".

En relación con la lectura, el trabajo de Spires et al. (2018) ofrece una síntesis de la literatura existente sobre las diferentes formas de leer, las estrategias utilizadas, las disposiciones con las que se aborda la construcción y la crítica de los sentidos de los textos y los usos diferenciados de esas interpretaciones que realizan los expertos en disciplinas académicas como la historia, las letras, la matemática o las ciencias naturales. Esos estudios muestran que los profesionales en actividad no sólo leen géneros diferentes, sino que realizan prácticas y seleccionan estrategias particulares. Como señala Di Capua-Hidalgo (2016b), la noción de género ha acompañado la evolución histórica de las prácticas periodísticas y las comunidades profesionales de la comunicación social poseen en la actualidad consolidadas tradiciones, prácticas y saberes científicos, impulsados, más recientemente, por la formación de nivel universitario en el área. Este trabajo tiene por objetivo realizar una descripción de esas prácticas en Uruguay, desde el tamiz de la lectura.

\section{Metodología}

\section{Instrumento de Recolección de los Datos}

La exploración de las prácticas lectoras podría abordarse a través de la observación desde una perspectiva etnográfica, del uso de los conocimientos previos de estudios relacionados aprovechando taxonomías preexistentes o de preguntas realizadas directamente a los actores. En este caso, debido a limitaciones de tiempo y a que el objetivo general de nuestra investigación es el diseño de un currículo organizado en torno a géneros discursivos y el abordaje de las prácticas lectoras de manera situada, elegimos un método de recolección de datos que proporcionara insumos de manera rápida para poder completar las siguientes fases del proyecto de investigación-acción.

La técnica utilizada para esta fase fue una encuesta que incluye datos cuantitativos y cualitativos recogidos a través de preguntas cerradas y abiertas, ya que brinda un panorama general del problema y también permite obtener información específica sobre las prácticas de un gran número de personas en la comunidad profesional de la comunicación. El objetivo de la encuesta fue determinar la diversidad de prácticas de literacidad profesional que se usan en una comunidad de profesionales del área de la comunicación en Uruguay.

La encuesta incluyó ítems deductivos e inductivos, es decir, un conjunto de tópicos, categorías y dimensiones definidos previamente, para reconocer cuáles eran identificados por el grupo, de acuerdo con su cuantificación numérica (JANSEN, 2010). La utilización de un número importante de ítems deductivos se justificó porque ellos nos permitían obtener, en un lapso acotado, un volumen importante 
de información, a fin de dar un primer paso de definición de las características del objeto de estudio, a partir de las cuales, organizar los pasos siguientes de la investigación.

El cuestionario diseñado incluyó preguntas demográficas y otras en las que se pidió a los participantes identificar las prácticas lectoras y los géneros que caracterizan sus actividades profesionales. La redacción de esos ítems se basó en la concepción de sistema de actividades y de sistema de géneros de Bazerman (2005), al indagar los géneros leídos y producidos, su secuencia, sus relaciones y sus finalidades. En cuanto a las prácticas y estrategias de lectura, tomamos como base el trabajo de Spires et al. (2018) sobre las prácticas de lectura en las humanidades y las ciencias naturales, considerando el carácter interdisciplinario de la profesión del comunicador e incorporando la exploración del uso de diferentes lenguas. En la comunidad profesional de la comunicación existe un debate sobre el estatus disciplinar del área. Por un lado, se sostiene que la comunicación constituye una disciplina en sí misma, ya que existen títulos y trabajos que se identifican dentro de ella, además de una tradición histórica de conocimiento acumulado. Por otro lado, se argumenta que esta comunidad profesional se caracteriza por su interdisciplinariedad, integrando diferentes disciplinas de las ciencias sociales y las humanidades en la construcción de conocimiento sobre un problema complejo como es la comunicación (CALHOUN, 2011). En nuestro contexto, la perspectiva interdisciplinar representa más cabalmente a la comunidad profesional dedicada a la comunicación ${ }^{3}$.

Los ítems relacionados con las prácticas lectoras, la selección de diferentes lenguas y las finalidades de la lectura fueron elaboradas por nuestro grupo de investigación, teniendo en cuenta las lenguas de uso más común en Uruguay, las modalidades de producción y comprensión de texto ${ }^{4}$ (producción de texto oral, producción de texto escrito, etc.) y las posibles finalidades profesionales de la lectura. El inventario de géneros fue realizado teniendo como base el propuesto por Dolz y Schneuwly (2004). Sin embargo, a esta lista fueron agregados géneros sugeridos por profesionales de la comunicación consultados, así como por los integrantes del equipo de investigación. Un ítem de respuesta cerrada incluye una escala Likert asociada con frecuencias (de muy frecuente a nada frecuente), mientras que el resto de los ítems de respuesta cerrada ofrecen opciones no excluyentes (por ejemplo, lenguas en las que se lee).

El cuestionario fue pre testeado por integrantes del grupo de investigación (5 profesores universitarios) y por 3 profesionales de la comunicación (un comunicador organizacional, un comunicador audiovisual y un periodista). Las devoluciones de esos participantes, recogidas en entrevistas particulares, permitieron corroborar la comprensión delos enunciados, agregaropciones no contempladas originalmente (finalidades para la lectura y géneros), simplificar varios ítems para hacerlos más comprensibles, y agregar a cada lista cerrada de opciones un campo para que el participante pudiese incorporar informaciones no contempladas (géneros, finalidades, estrategias, etc.).

\footnotetext{
${ }^{3}$ De acuerdo a la teoría de códigos de Bernstein (2000), se pueden distinguir los tipos de construcción de conocimiento de las disciplinas. Podemos diferenciar entre estructuras de conocimiento que distinguen formas situadas y locales de construcción de conocimiento, de otras especializadas y definidas de manera que pueden relacionarse significados entre instancias particulares a lo largo del tiempo. Más recientemente, la teoría de legitimación de códigos de Karl Maton (2013) extiende esta perspectiva y dialoga junto con la Lingüística Sistémico-Funcional para caracterizar las formas en que se construye conocimiento a través de diferentes configuraciones de prácticas, cosmologías y opciones semánticas en áreas de conocimiento, como las ciencias duras o las humanidades. En el caso de la comunicación, la caracterización del área correspondería a estructuras más situadas y a una densidad semántica más débil debido a la diversidad y heterogeneidad de las prácticas de construcción de conocimiento en esta comunidad profesional.

${ }^{4}$ Para la redacción de las tres opciones del ítem referente a los propósitos de la lectura (leer para producir un texto escrito, leer para producir un texto oral, leer para producir un texto multimodal), definimos texto escrito como aquel en que predomina una semiosis lingüística gráfica, texto oral como aquel en que predomina una semiosis lingüística sonora y texto multimodal como aquel texto en que ninguna de estas semiosis predomina y que contiene, además, otras formas de generar significado, principalmente por medios audiovisuales. Para esta definición tenemos como base los conceptos de Marcuschi (2007) relativos al continuo oralidad/escritura/otras formas semióticas, en que ocurren los textos de los diferentes géneros. La simplicidad de nuestra categorización no obedeció a una intención de simplificar la teoría al respecto, sino al interés por ofrecer una taxonomía inteligible para los participantes.
} 
Para indagar la secuencia de uso (lectura y producción) de los textos de cada género, recurrimos a una pregunta abierta (“ ¿Recuerdas si, en tu práctica profesional, asocias la lectura de alguno de los géneros de la pregunta anterior con la producción de algún otro género específico? Por ejemplo: ‘Sí, he leído novelas para escribir un guión' o 'Sí, leo normas jurídicas para escribir noticias”'). Finalmente, las prácticas de lectura y estrategias fueron formuladas en base a las propuestas por Spires et al. (2018).

Las respuestas a las 7 preguntas del cuestionario (6 cerradas y 1 abierta) fueron recogidas usando la herramienta de Cuestionario de Google, enviada por e-mail a partir de una base de datos inicial, que fue ampliada con contactos sugeridos, progresivamente, por los participantes (técnica de bola de nieve).

\section{Construcción de la Muestra}

Con el objetivo de recoger los datos a partir de un grupo que tuviera las características sobresalientes que inciden en las prácticas lectoras de esta comunidad profesional (symbolic representation) (RITCHIE; LEWIS; ELAM, 2003), garantizando la suficiente diversidad de perfiles (saturación) (RITCHIE; LEWIS; ELAM, 2003; JANSEN, 2010), los participantes fueron elegidos intencionalmente (purposing sample) (RITCHIE; LEWIS; ELAM, 2003). Buscamos obtener un grupo de egresados que actuaran en diferentes áreas (comunicación organizacional, comunicación educativa y comunitaria, periodismo, publicidad, audiovisual, multimedia y tic, docencia, gestión cultural e investigación), teniendo como referencia una encuesta realizada a egresados de nuestra facultad (URUGUAY, 2016). Esta encuesta funcionó como referencia de muestra (sample frame) (RITCHIE; LEWIS; ELAM, 2003), por incluir una descripción de las principales características de la población general de egresados, lo que nos proveyó criterios de selección para la elaboración de una muestra balanceada (balanced sample) (RITCHIE; LEWIS; ELAM, 2003).

El Gráfico 1 muestra las proporciones similares en la composición de ambos grupos, el de la muestra de referencia y el de la muestra de este estudio. Los valores no son iguales debido a que esa encuesta permitía a los participantes identificarse con más de un área profesional, mientras que en nuestro cuestionario los participantes debían elegir el área predominante de su actuación profesional a fin de especificar el análisis por área en futuros estudios.

Sin embargo, para balancear ambas muestras, buscamos que el área predominante en este estudio fuera también la comunicación organizacional, como en la muestra de referencia, es decir, que duplicara a cada una de las áreas del segundo grupo más numeroso (periodismo, comunicación educativa y comunitaria, audiovisual y publicidad). A su vez, se buscó que las áreas que ocupan el tercer grupo numérico, en la muestra de referencia (docencia, investigación y gestión cultural), tuvieran la misma proporción en la muestra de este estudio. Excluimos de esta exigencia al área Multimedia y Tic, por considerar que ella se encontraba incluida en la identificación de los participantes de otras áreas (publicidad, audiovisual, comunicación organizacional, gestión cultural).

De esa forma, la diversidad de los participantes permitió constituir una muestra que representara aspectos importantes en la constitución de las prácticas profesionales estudiadas (RITCHIE; LEWIS; ELAM, 2003). Siguiendo esos criterios, al llegar a los 82 participantes, obtuvimos una muestra con proporciones relativamente similares a las de la muestra de referencia.

También se tuvo como segundo criterio de balanceo de la muestra incluir participantes con trayectorias profesionales de diferente duración (hasta 5 años, entre 5 y 10 años, y más de 10 años). La Tabla 1 muestra la proporción similar de participantes de cada grupo en la muestra de este estudio. 
Tabla 1: Trayectorias de ejercicio profesional de los participantes por años. Fuente: los autores

\begin{tabular}{|c|c|}
\hline \multicolumn{2}{|c|}{$¿$ Cuánto tiempo ha ejercido la profesión en esta área? } \\
\hline Hasta 5 años & $25,6 \%(11 / 82)$ \\
\hline Entre 5 y 10 años & $36,6 \%(30 / 82)$ \\
\hline Más de 10 años & $37,8 \%(31 / 82)$ \\
\hline
\end{tabular}

Fuente: Elaboración propia.

Gráfico 1. Áreas profesionales de los participantes de la encuesta a egresados en comunicación (URUGUAY, 2016) y de la encuesta analizada en este artículo. Fuente: los autores

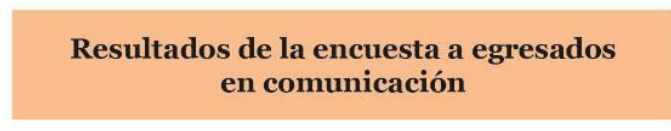

¿Con cuál/es áreas de la comunicación considera que está relacionado su trabajo actual?

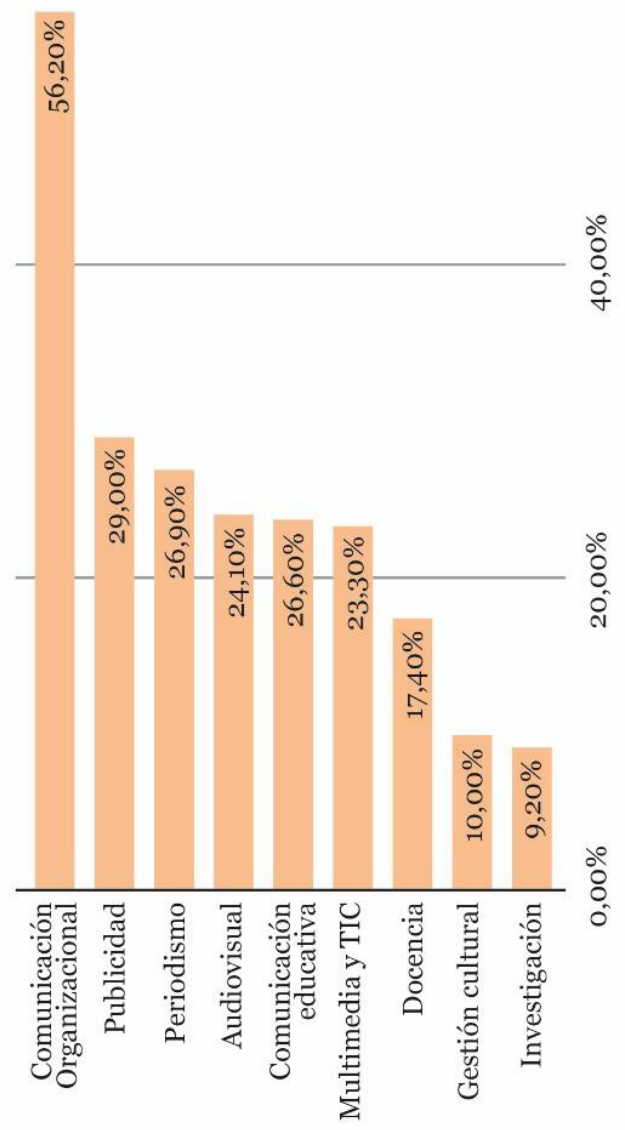

\section{Resultados de la encuesta sobre lectura a profesionales de la comunicación}

Seleccione la orientación profesional dentro de la que desempeña actualmente su principal labor como comincador/a

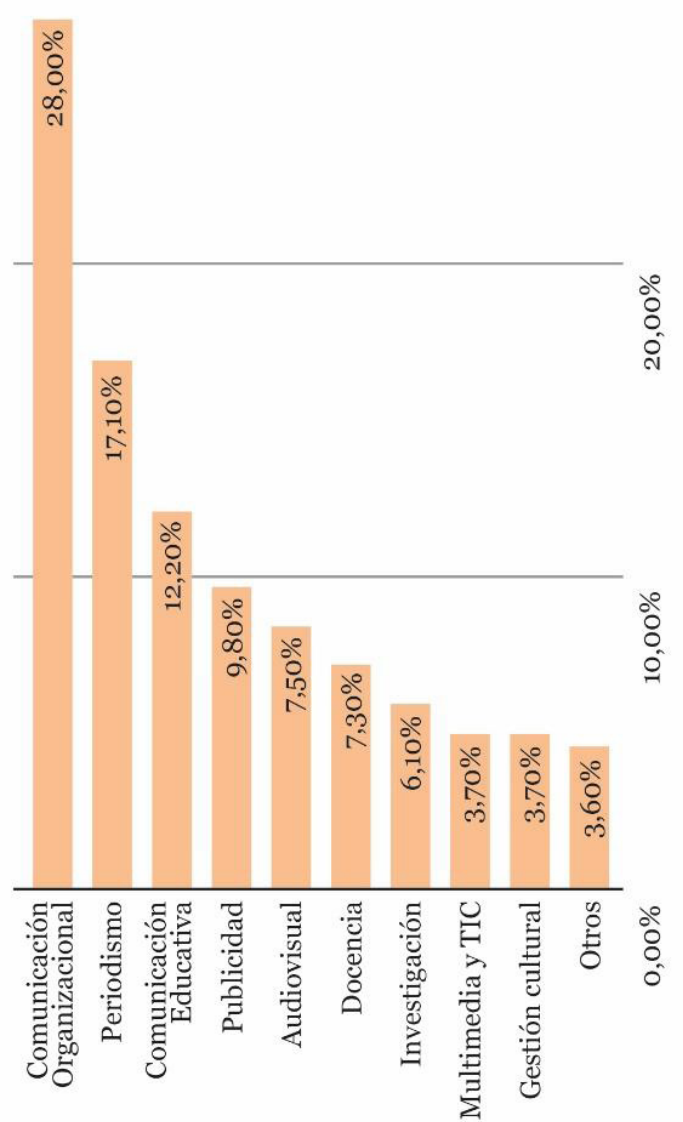

Fuente: Elaboración propia.

\section{Tratamiento de los Datos}

Los datos fueron analizados siguiendo criterios cuantitativos y cualitativos de recurrencia y proporcionalidad, buscando reconocer aspectos destacados y marginales de las prácticas del grupo (JANSEN, 2010). Como se trata de una primera exploración, el análisis de este artículo buscó reconocer, por medio de descripciones numéricas de naturaleza descriptiva, las prácticas presentes en el grupo en su totalidad, dejando 
para etapas posteriores el análisis basado en las áreas profesionales y en duración de la trayectoria profesional. Para analizar las respuestas a la pregunta abierta, fueron sintetizadas las descripciones aportadas por cada participante, considerando las acciones sociales/profesionales y de lenguaje (escribir una noticia, realizar una exposición oral) y los géneros mencionados (leídos y escritos). Esa síntesis permitió crear categorías recurrentes y establecer su proporción dentro de la totalidad de las respuestas. La codificación fue realizada por dos de los autores y verificada para asegurar la consistencia en la categorización.

\section{Resultados}

\section{Prácticas y Propósitos de Lectura en la Profesión: ¿en qué lenguas leen y para qué?}

Los resultados muestran en qué lenguas los participantes saben leer y en qué lenguas, incluyendo la L1, realizan lecturas relacionadas con la profesión. La lengua principal de lectura es el español, aunque el inglés y el portugués también ocupan lugares importantes (Tabla 2).

Tabla 2: ¿En qué lenguas extranjeras pueden leer y leen? Fuente: los autores

\begin{tabular}{|c|c|c|c|}
\hline $\begin{array}{c}\text { Indique las lenguas extranjeras en las que usted puede } \\
\text { leer textos }\end{array}$ & $\begin{array}{c}\text { Indique las lenguas en las que usted lee textos para } \\
\text { práctica profesional }\end{array}$ \\
\hline Inglés & $92,7 \%(76 / 82)$ & Español & $96,3 \%(79 / 82)$ \\
\hline Portugués & $70,7 \%(58 / 82)$ & Inglés & $72 \%(59 / 82)$ \\
\hline Italiano & $23,2 \%(19 / 82)$ & Portugués & $39 \%(32 / 82)$ \\
\hline Francés & $2,4 \%(2 / 82)$ & Italiano & $4,9 \%(4 / 82)$ \\
\hline Alemán & $1,2 \%(1 / 82)$ & Francés & $3,7 \%(3 / 82)$ \\
\hline Catalán & $1,2 \%(1 / 82)$ & Catalán & $1,2 \%(1 / 82)$ \\
\hline Otras & $2,4 \%(2 / 82)$ & Alemán & $0 \%(0 / 82)$ \\
\hline
\end{tabular}

Fuente: Elaboración propia.

La acción profesional más recurrente es leer para escribir, seguida, en frecuencias similares, por leer como actividad no relacionada a la producción. Luego, leer para preparar textos orales, o multimodales, son menos frecuentes (Tabla 3).

Tabla 3: Frecuencia de los propósitos de lectura. Fuente: los autores

Leer un texto escrito en la preparación de otro texto escrito (noticia, guion, informe, etc.).

\begin{tabular}{|l|l|}
\hline Muy frecuente & $62,2 \%(51 / 82)$ \\
\hline Algo frecuente & $20,7 \%(17 / 82)$ \\
\hline Frecuente & $13,4 \%(11 / 82)$ \\
\hline Poco frecuente & $2,4 \%(2 / 82)$ \\
\hline Nada frecuente & $1,2 \%(1 / 82)$ \\
\hline
\end{tabular}

Leer uno o más textos escritos sin que ello conlleve, necesariamente, a la producción de otro texto oral o escrito.

\begin{tabular}{|l|l|}
\hline Muy frecuente & $46,3 \%(38 / 82)$ \\
\hline Algo frecuente & $20,7 \%(17 / 82)$ \\
\hline Frecuente & $17,1 \%(14 / 82)$ \\
\hline Poco frecuente & $8,5 \%(7 / 82)$ \\
\hline Nada frecuente & $7,3 \%(6 / 82)$ \\
\hline
\end{tabular}


Leer un texto escrito en la preparación de otro texto oral (presentación, reunión, conferencia, clase, etc.).

\begin{tabular}{|l|l|}
\hline Muy frecuente & $40,2 \%(33 / 82)$ \\
\hline Algo frecuente & $17,1 \%(14 / 82)$ \\
\hline Frecuente & $13,4 \%(11 / 82)$ \\
\hline Poco frecuente & $12,2 \%(10 / 82)$ \\
\hline Nada frecuente & $17,1 \%(14 / 82)$ \\
\hline
\end{tabular}

Leer un texto escrito en la preparación de otro texto multimodal (pieza radial, pieza audiovisual, pieza publicitaria).

\begin{tabular}{|l|l|}
\hline Muy frecuente & $22,0 \%(18 / 82)$ \\
\hline Algo frecuente & $25,6 \%(21 / 82)$ \\
\hline Frecuente & $25,6 \%(21 / 82)$ \\
\hline Poco frecuente & $11,0 \%(9 / 82)$ \\
\hline Nada frecuente & $15,9 \%(13 / 82)$ \\
\hline
\end{tabular}

Fuente: Elaboración propia.

En cuanto a los propósitos específicos de lectura, en un primer nivel (del 80\% al 100\% de los participantes) está la lectura para la búsqueda de fuentes de información y para la búsqueda de información de naturaleza teórica (1 y 2 de la Tabla 4). En un segundo nivel (60\% a 80\%), se encuentran la ampliación de un concepto teórico o su mayor comprensión, la búsqueda de información general sobre una temática o del contexto de un hecho, la búsqueda de más de un punto de vista sobre un tema específico, y la obtención de información específica necesaria en instrucciones o bases de concursos para la elaboración de un proyecto (4 a 8 de la Tabla 4). Finalmente, con menor recurrencia (40\% al 60\%), encontramos la búsqueda de secciones específicas para citar, la búsqueda de información para verificación teórica, la búsqueda de modelos de textos y la búsqueda de ejemplos de hechos específicos (9 a 13 de la Tabla 4).

Tabla 4: Propósitos específicos de la lectura. Fuente: los autores

\begin{tabular}{|l|l|}
\hline $\begin{array}{l}\text { Marque las opciones de la columna que Sí se correspondan con motivos por los cual usted LEE UN TEXTO } \\
\text { ESCRITO en su práctica profesional. }\end{array}$ \\
\hline 1 - Para obtener información como fuente para escribir otro texto (oral o escrito) & $87,8 \%(72 / 82)$ \\
\hline 2 - Para usarlo como referencia teórica para producir otro texto & $80,5 \%(60 / 82)$ \\
\hline 3 - Para ampliar un concepto leído en otro texto escrito & $78 \%(64 / 82)$ \\
\hline 4 - Para entender un concepto específico que se quiere utilizar en otro texto (oral o escrito) & $78 \%(64 / 82)$ \\
\hline $\begin{array}{l}5 \text { - Para obtener información general sobre una temática sobre la que se debe producir (en forma oral o } \\
\text { escrita) }\end{array}$ & $76,8 \%(63 / 82)$ \\
\hline $\begin{array}{l}\text { 6 - Para obtener información sobre el contexto de un hecho sobre el que se debe producir (en forma oral } \\
\text { o escrita) }\end{array}$ & $70,7 \%(58 / 82)$ \\
\hline $\begin{array}{l}7 \text { - Para obtener más de un punto de vista sobre un tema específico sobre el que se debe producir (en } \\
\text { forma oral o escrita) }\end{array}$ & $67,1 \%(55 / 82)$ \\
\hline $\begin{array}{l}\text { 8 - Para obtener información (en instrucciones o bases) necesaria para la elaboración (oral o escrita) de } \\
\text { un proyecto }\end{array}$ & $63,4 \%(52 / 82)$ \\
\hline 9 - Para buscar secciones específicas para citar en otro texto & $51,2 \%(42 / 82)$ \\
\hline 10 - Para verificar la validez de la afirmación de un autor en otro texto & $51,2 \%(42 / 82)$ \\
\hline $\begin{array}{l}11 \text { - Para tomarlo como modelo para escribir otro texto } \\
\text { 12 - Para buscar ejemplos de hechos a reseñar en otro texto }\end{array}$ & $48,8 \%(40 / 82)$ \\
\hline $\begin{array}{l}13 \text { - Para obtener información sobre la forma de pensar de una persona en particular y reflejarla en un } \\
\text { texto (oral o escrito) }\end{array}$ & $45,1 \%(37 / 82)$ \\
\hline 14 - Otros & $40,2 \%(33 / 82)$ \\
\hline
\end{tabular}

Fuente: Elaboración propia. 
Por lo tanto, las prácticas profesionales más frecuentes son leer en español o en otras lenguas (inglés y portugués), en relación muy frecuente con la escritura, y con la finalidad de, principalmente, encontrar información general sobre hechos y su contexto o acceder a información teórica general sobre ciertas temáticas.

\section{Prácticas y Géneros: ¿̨leen qué para producir qué?}

El análisis desde el tamiz de los géneros nos permite reconocer lecturas de géneros de la esfera periodística como la noticia y la entrevista a una figura pública (80\% a 100\%; 1 y 2 de la Tabla 5). En el nivel siguiente $(60 \%$ a $80 \%)$, se encuentran géneros de la misma esfera (entrevista a un especialista, artículo de opinión, reportaje y editorial), de la esfera académica (capítulo de libro, capítulo de libro académico, artículo científico), y los informes técnicos, que pueden corresponder a diferentes esferas de actividad (3 a 10 de la Tabla 5). En una tercera franja (40\% a 60\%), se encuentran géneros de diversas esferas: convocatorias a concurso, ensayos, normas jurídicas, reseña crítica, artículo de divulgación, cuento y novela (11 a 17 de la Tabla 5). Finalmente, biografía, libro académico completo, crónica literaria, poema y pieza teatral fueron seleccionadas por un número menor de participantes (menos del 40\%) (18 a 22 de la Tabla 5).

Tabla 5: Géneros leídos en la práctica profesional. Fuente: los autores

\begin{tabular}{|c|c|}
\hline 1 - Noticia publicada en un medio de comunicación escrito & $86,6 \%(71 / 82)$ \\
\hline 2 - Entrevista a una figura pública publicada en un medio de comunicación escrito & $81,7 \%(67 / 82)$ \\
\hline 3 - Entrevista a un especialista en una temática publicada en un medio de comunicación escrito & $76,8 \%(63 / 82)$ \\
\hline 4 - Capítulo de un libro escrito por un determinado autor o autores & $73,2 \%(60 / 82)$ \\
\hline 5 - Artículo o columna de opinión publicada en un medio de comunicación escrito & $70,7 \%(58 / 82)$ \\
\hline 6 - Capítulo de un libro académico escrito por varias personas & $70,7 \%(58 / 82)$ \\
\hline 7 - Informe técnico & $69,5 \%(57 / 82)$ \\
\hline 8 - Artículo científico publicado en una revista especializada & $65,9 \%(54 / 82)$ \\
\hline 9 - Reportaje extenso & $64,6 \%(53 / 82)$ \\
\hline 10 - Editorial publicado en un medio de comunicación escrito & $62,2 \%(51 / 82)$ \\
\hline 11 - Convocatorias y bases para financiación & $57,3 \%(47 / 82)$ \\
\hline 12 - Ensayo académico & $57,3 \%(47 / 82)$ \\
\hline 13 - Normas jurídicas & $52,4 \%(43 / 82)$ \\
\hline 14 - Reseña crítica publicada en un medio de comunicación escrito & $52,4 \%(43 / 82)$ \\
\hline 15 - Artículo de divulgación científica & $40,0 \%(33 / 82)$ \\
\hline $16-$ Cuento & $40,2 \%(33 / 82)$ \\
\hline 17 - Novela & $40,2 \%(33 / 82)$ \\
\hline 18 - Biografía, autobiografía & $35,4 \%(29 / 82)$ \\
\hline 19 - Libro académico completo escrito por un determinado autor o autores & $35,4 \%(29 / 82)$ \\
\hline 20 - Crónica literaria & $25,6 \%(21 / 82)$ \\
\hline 21 - Poema & $18,3 \%(15 / 82)$ \\
\hline 22 - Pieza teatral & $7,3 \%(6 / 82)$ \\
\hline $23-$ Otros & $1,2 \%(1 / 82)$ \\
\hline
\end{tabular}

Fuente: Elaboración propia.

La información sobre los géneros asociados en secuencia en la lectura y en la producción (que se leen para producir otro texto) fue recogida por medio de una pregunta de respuesta abierta que resultó en un total de 59 descripciones de prácticas profesionales (Ej.: "Leo noticias para generar informes 
de diagnóstico económico y social”). Las respuestas fueron categorizadas agrupando los géneros asociados con la lectura y con la producción dentro de categorías no excluyentes, ya que la mayoría de los participantes hacían referencia a leer varios géneros en sus respuestas y en cantidades diferentes. Los términos utilizados por los participantes para referirse a los géneros, en su mayoría, reflejan los utilizados en el ítem anterior, aunque nomenclaturas diferentes surgieron y fueron agrupadas bajo un término común (Ej.: "exposiciones orales”, “textos orales”, “textos orales de clases”, "presentaciones en el ámbito laboral"). Esta coincidencia con relación a las nomenclaturas utilizadas para designar a los géneros supone la necesidad de un trabajo cualitativo posterior, basado en entrevistas semiestructuradas, que permita confirmar los términos más utilizados por los profesionales para definir los géneros que forman parte de sus prácticas, ya que estas selecciones léxicas podrían deberse a un efecto de priming.

Esa categorización reafirma, en gran medida, las observaciones anteriores en relación a la vitalidad de la lectura de textos de la esfera periodística y académica (Tabla 6), pues los géneros de lectura más mencionados (más de 10 menciones en el total) son de las esferas periodística (noticias y entrevistas) y académica (artículos científicos y textos académicos), aunque también se destacan las normas jurídicas (esfera jurídica y política) y los informes técnicos (diversas esferas técnico-profesionales).

Tabla 6: Secuencia de lectura y producción de géneros. Fuente: los autores

\begin{tabular}{|c|c|}
\hline LECTURA & PRODUCCIÓN \\
\hline Normas jurídicas $34 \%$ (20/59) & $\begin{array}{l}\text { Noticias } 50 \%(10 / 20) \\
\text { Proyectos } 10 \%(2 / 20) \\
\text { Artículos científicos } 10 \%(2 / 20) \\
\text { Normas jurídicas } 5 \%(1 / 20) \\
\text { Notas en redes sociales } 5 \%(1 / 20) \\
\text { Planes de trabajo } 5 \%(1 / 20) \\
\text { Exposiciones orales } 5 \%(1 / 20) \\
\text { Ensayos } 5 \%(1 / 20) \\
\text { Entrevistas } 5 \%(1 / 20)\end{array}$ \\
\hline Noticias 32\% (19/59) & $\begin{array}{l}\text { Noticias } 26 \%(5 / 19) \\
\text { Exposiciones orales } 16 \%(3 / 19) \\
\text { Guiones } 16 \%(3 / 19) \\
\text { Piezas de comunicación visual } 11 \%(2 / 19) \\
\text { Proyectos } 11 \%(2 / 19) \\
\text { Informes técnicos } 5 \%(1 / 19) \\
\text { Entrevistas } 5 \%(1 / 19) \\
\text { Planes de trabajo } 5 \%(1 / 19) \\
\text { Artículos científicos 5\% }(1 / 19)\end{array}$ \\
\hline Informes técnicos $31 \%(18 / 59)$ & $\begin{array}{l}\text { Noticias } 56 \%(10 / 18) \\
\text { Comunicados } 11 \%(2 / 18) \\
\text { Exposiciones orales } 11 \%(2 / 18) \\
\text { Guiones } 6 \%(1 / 18) \\
\text { Nota en redes sociales } 6 \%(1 / 18) \\
\text { Presentaciones audiovisuales } 6 \%(1 / 18) \\
\text { Contenidos gráficos } 6 \%(1 / 18)\end{array}$ \\
\hline Artículos científicos 25\% (15/59) & $\begin{array}{l}\text { Noticias } 27 \%(4 / 15) \\
\text { Entrevistas } 13 \%(2 / 15) \\
\text { Exposiciones orales } 13 \%(2 / 15) \\
\text { Guiones } 7 \%(1 / 15) \\
\text { Proyectos } 7 \%(1 / 15) \\
\text { Artículos científicos } 7 \%(1 / 15) \\
\text { Investigaciones periodísticas } 7 \%(1 / 15) \\
\text { Artículos de opinión } 7 \%(1 / 15) \\
\text { Notas de divulgación científica } 7 \%(1 / 15) \\
\text { Planes de trabajo } 7 \%(1 / 15)\end{array}$ \\
\hline
\end{tabular}




\begin{tabular}{|c|c|}
\hline Entrevistas $20 \%(12 / 59)$ & $\begin{array}{l}\text { Noticias } 42 \%(5 / 12) \\
\text { Entrevistas } 17 \%(2 / 12) \\
\text { Guiones } 8 \%(1 / 12) \\
\text { Piezas publicitarias } 8 \%(1 / 12) \\
\text { Exposiciones orales } 8 \%(1 / 12) \\
\text { Informes institucionales } 8 \%(1 / 12) \\
\text { Planes de trabajo } 8 \%(1 / 12)\end{array}$ \\
\hline Textos académicos 19\% $(11 / 59)$ & $\begin{array}{l}\text { Noticias } 27 \%(3 / 11) \\
\text { Exposiciones orales } 27 \%(3 / 11) \\
\text { Guiones } 9 \%(1 / 11) \\
\text { Investigaciones periodísticas } 9 \%(1 / 11) \\
\text { Artículos de opinión } 9 \%(1 / 11) \\
\text { Entrevistas } 9 \%(1 / 11) \\
\text { Proyectos } 9 \%(1 / 11)\end{array}$ \\
\hline
\end{tabular}

Fuente: Elaboración propia.

El género más común en la producción es la noticia, es decir, muchos participantes leen noticias, entrevistas, normas jurídicas, informes técnicos y artículos científicos para escribir noticias, sea dentro del área del periodismo, de la publicidad, etc. Una explicación posible a la vitalidad de este género es su presencia en la práctica profesional de los comunicadores de las dos mayores áreas de actividad profesional de los participantes (comunicación institucional y periodismo), aunque un estudio diferenciado de la relación entre las áreas profesionales y los diferentes géneros de lectura y producción aún debe ser realizado en mayor profundidad. Un segundo género de producción destacable es la exposición oral, hecho que también se explica por su utilidad en todas las áreas profesionales, aspecto que destaca el papel relevante que la lectura para la producción oral podría ocupar en la enseñanza de la lectura. Por último, como sugería la Tabla 2, los participantes centran sus lecturas en las esferas periodística, técnicas y académica, con la finalidad de producir géneros propios de sus esferas de actuación profesional (noticias, entrevistas, exposiciones orales, guiones, piezas de comunicación audiovisual, proyectos, etc.). Resta profundizar, en estudios posteriores, la capacidad explicativa de este hallazgo para las prácticas de los profesionales de todas las áreas de la comunicación en forma específica, es decir, si en todas ellas la lectura gira principalmente en torno de géneros periodísticos, técnicos y académicos.

Las menciones a la lectura y a la producción de ciertos géneros nos muestran que los participantes leen textos/géneros de la misma esfera de actividad en la que se desempeñan, es decir, la esfera periodística o de la comunicación social, y complementan esas lecturas, principalmente, con textos/géneros del ámbito académico y profesional. Esto coincide con lo que destacamos en la sección anterior, respecto de las finalidades de la lectura, es decir, los comunicadores leen para buscar fuentes de información, conceptos teóricos y para obtener información general sobre una temática o sobre el contexto de un hecho. Podemos suponer que las fuentes de información específica sobre hechos y contextos sean encontradas en géneros de la esfera periodística, y que los géneros provenientes del ámbito académico y profesional sirvan para ampliar la comprensión teórica o técnica de los fenómenos sobre los que se pretende producir.

Es necesario continuar investigando para comprender el papel importante que la lectura de textos de la esfera académica ocupa en las prácticas de la comunicación. Es posible que la presencia de estos géneros se deba a su importancia en el ámbito de la formación académica, en la que, como constata Di Capua-Hidalgo (2016a) para el caso chileno, los estudiantes se forman en contacto con géneros tanto del ámbito profesional de la comunicación como del ámbito académico. También es necesario conocer las motivaciones profesionales más concretas la lectura de textos académicos. 


\section{Prácticas Letradas y Estrategias Específicas: ¿qué hacen cuando leen?}

El análisis de las prácticas letradas y estrategias muestra que el primer grupo más seleccionado $(60 \%$ a $80 \%$ ) se compone de operaciones como sintetizar lo leído, relacionar el contenido con el contexto histórico, detectar la terminología técnica, evaluar la confiabilidad de las fuentes, analizar el material gráfico, evaluar la calidad de las afirmaciones, contrastar las visiones del texto y jerarquizar los contenidos más relevantes (1 a 9 de la Tabla 7). Todas esas operaciones son recogidas por Spires et al. (2018) como estrategias de lectura en las disciplinas científicas, especialmente ciencias, matemática e historia. La mayoría de estas estrategias suponen un abordaje crítico de los textos, lo que podría suponer que esa sea una característica de la literacidad de este grupo. Conocer las motivaciones sociales que llevan a estos lectores a una evaluación crítica de los textos es un aspecto que futuras investigaciones deben abordar.

En segundo lugar (40\% a 60\%), se encuentran contextualizar el texto por su autor, relacionarlo con la experiencia personal y con el ámbito de la disciplina científica en la que está inserto el texto, evaluar la coherencia lógica de sus argumentaciones y la forma en que las ideas son expresadas por medio de figuras de lenguaje, evaluar la validez científica de sus afirmaciones y el prestigio de los autores citados en el texto, relacionar el texto con el medio de publicación, analizar su organización, sintetizar la posición explícita e implícita del autor y las consecuencias posibles de sus afirmaciones (10 a 21 de la Tabla 7). En último lugar (hasta 40\%), se encuentran operaciones como analizar las voces del texto, evaluar la relación entre los objetivos de un texto y sus conceptos, su coherencia temporal, el alcance de sus afirmaciones, la adecuación a su género y el valor poético de algunas de sus expresiones (22 a 27 de la Tabla 7). Este último análisis presenta una interrogante respecto de la poca importancia que las estrategias de análisis de la forma de un texto parecen tener en las prácticas profesionales, considerando que el lenguaje verbal no ocupa el mismo lugar como medio de construcción de sentido para los profesionales de las diferentes áreas. Piénsese, por ejemplo, en las diferencias entre las prácticas de periodistas y realizadores audiovisuales.

Tabla 7: Prácticas y estrategias de lectura. Fuente: los autores

\begin{tabular}{|l|l|}
\hline $\begin{array}{l}\text { Marque las opciones de la columna que SÍ se correspondan con estrategias de lectura que usted realiza al leer } \\
\text { textos escritos en su práctica profesional. }\end{array}$ \\
\hline 1 - Sintetizar mentalmente los principales conceptos o ideas del texto & $78 \%(64 / 82)$ \\
\hline 2 - Relacionar los contenidos del texto con el contexto social y el momento histórico en que fue publicado & $68.3 \%(56 / 82)$ \\
\hline 3 - Analizar, destacar, investigar terminología técnica contenida en el texto & $65.9 \%(54 / 82)$ \\
\hline 4 - Analizar la confiabilidad de las fuentes presentadas en el texto & $65.9 \%(54 / 82)$ \\
\hline 5 - Analizar informaciones contenidas en gráficos, figuras o cuadros del texto & $64.6 \%(53 / 82)$ \\
\hline 6 - Analizar fotografías o imágenes que acompañan el texto & $63.4 \%(52 / 82)$ \\
\hline 7 - Analizar la calidad (precisión, alcance, utilidad) de los conceptos contenidos en el texto & $62.2 \%(51 / 82)$ \\
\hline $\begin{array}{l}8-\text { Relacionar mentalmente los principales conceptos del texto con posiciones diferentes, propias o } \\
\text { de otros }\end{array}$ & $61 \%(50 / 82)$ \\
\hline 9 - Analizar segmentos que contengan contenidos relevantes del texto & $61 \%(50 / 82)$ \\
\hline 10 - Relacionar los contenidos del texto con el papel social o institucional del autor o con su historia. & $59.8 \%(49 / 82)$ \\
\hline 11 - Relacionar los contenidos del texto con su experiencia personal en la temática & $57.3 \%(47 / 82)$ \\
\hline 12 - Relacionar los contenidos del texto con el ámbito de la disciplina científica en la que está inserto & $54.9 \%(45 / 82)$ \\
\hline 13 - Analizar la coherencia lógica de las argumentaciones contenidas en el texto & $52.4 \%(43 / 82)$ \\
\hline $\begin{array}{l}14 \text { - Analizar la forma en que las ideas son expresadas por medio de figuras de lenguaje o términos con } \\
\text { potente valor expresivo }\end{array}$ & $51.2 \%(42 / 82)$ \\
\hline $\begin{array}{l}15 \text { - Analizar la validez de las afirmaciones contenidas en el texto de acuerdo con las evidencias que } \\
\text { presenta }\end{array}$ & $51.2 \%(42 / 82)$ \\
\hline 16 - Analizar la calidad o prestigio de los autores citados en el texto & $51.2 \%(42 / 82)$ \\
\hline
\end{tabular}




\begin{tabular}{|l|l|}
\hline $\begin{array}{l}17 \text { - Relacionar los contenidos del texto con el papel social o institucional del medio de publicación o } \\
\text { con su historia }\end{array}$ & $50 \%(41 / 82)$ \\
\hline 18 - Analizar la forma en que los contenidos están organizados a lo largo del texto & $47.6 \%(39 / 82)$ \\
\hline 19 - Analizar y sintetizar la posición explícita del autor sobre los contenidos del texto & $45.1 \%(37 / 82)$ \\
\hline 20 - Analizar y sintetizar la posición implícita del autor sobre los contenidos del texto & $43.9 \%(36 / 82)$ \\
\hline 21 - Analizar las consecuencias posibles de las afirmaciones contenidas en el texto & $42.7 \%(35 / 82)$ \\
\hline 22 - Analizar las diferentes voces o personajes que son mencionados en el texto & $35.4 \%(29 / 82)$ \\
\hline $\begin{array}{l}23 \text { - Analizar la adecuación de los conceptos que el texto contiene a la forma como se presentan o a } \\
\text { sus objetivos }\end{array}$ & $35.4 \%(29 / 82)$ \\
\hline 24 - Analizar la coherencia temporal de las narraciones contenidas en el texto & $34.1 \%(28 / 82)$ \\
\hline $\begin{array}{l}25 \text { - Analizar el alcance o fuerza de las afirmaciones contenidas en el texto de acuerdo con como están } \\
\text { expresadas }\end{array}$ & $34.1 \%(28 / 82)$ \\
\hline 26 - Analizar la adecuación del texto a lo que se esperaría por su género & $31.7 \%(26 / 82)$ \\
\hline 27 - Analizar el valor poético de expresiones de segmentos del texto & $31.7 \%(26 / 82)$ \\
\hline
\end{tabular}

Fuente: Elaboración propia.

Las estrategias y prácticas disciplinares más comunes parecen coincidir con el perfil general de actividad trazado anteriormente, que suponía que la mayoría de los participantes lee géneros de las esferas periodística y académica para la escritura, y en la búsqueda de información sobre hechos específicos o referencias teóricas generales. Algunas de las estrategias más seleccionadas parecen adecuarse a la comprensión de textos de ambas esferas: sintetizar lo leído, relacionarlo con el contexto histórico, contrastar las visiones del texto y jerarquizar los contenidos más relevantes. En contraste, otras parecen asociarse más a la lectura de los géneros de la esfera periodística: evaluar la confiabilidad de las fuentes, analizar el material gráfico; y otras parecen más factibles como apoyo a la lectura de los géneros de la esfera académica: detectar la terminología técnica y evaluar la calidad de las afirmaciones. Las operaciones del segundo nivel también coinciden con este análisis, con especial énfasis para aquellas que parecen más relevantes para el abordaje de textos/géneros de la esfera académica: contextualizar el texto por su autor, relacionarlo con la experiencia personal y con el ámbito de la disciplina científica, evaluar la coherencia lógica de sus argumentaciones, evaluar la validez científica de sus afirmaciones y el prestigio de los autores citados en el texto. Finalmente, resaltamos que las prácticas que implican un análisis lingüístico y discursivo más detallados, y que se asocian a los profesionales de las letras (SPIRES et al., 2018), fueron menos seleccionadas: analizar las voces del texto, evaluar la relación entre objetivos de un texto y sus conceptos, su coherencia temporal, la adecuación a su género y el valor poético de sus expresiones.

Los resultados de la encuesta permiten esbozar un modelo potencial de sistema de actividades (con finalidad didáctica) en el que los profesionales de la comunicación leen géneros de la esfera periodística para buscar fuentes de información general sobre una temática o sobre su contexto, y géneros académicos para ampliar la comprensión teórica de los fenómenos. Este perfil se refleja en las operaciones de lectura más frecuentes, que contribuyen a interpretar esos textos de la esfera periodística (contextualizarlos históricamente, extraer su orientación, analizar su material gráfico y la confiabilidad de sus fuentes, etc.) y de la esfera académica (analizar su prestigio institucional, los autores citados, la calidad de sus evidencias o de sus argumentaciones, etc.) (Tabla 8). 
Tabla 8. Modelo de sistema de actividades y lectura. Fuente: los autores

\begin{tabular}{|c|c|c|c|c|c|}
\hline \multicolumn{4}{|l|}{ LECTURA } & \multicolumn{2}{|c|}{ PRODUCCIÓN } \\
\hline Género & Esfera & Estrategias & Propósitos & Modalidad & Género \\
\hline $\begin{array}{l}\text { Noticia } \\
\text { Entrevista } \\
\text { Artículo de } \\
\text { opinión Editorial } \\
\text { Reportaje }\end{array}$ & Periodística & $\begin{array}{l}\text { Para la } \\
\text { comprensión y } \\
\text { crítica de géneros } \\
\text { de la esfera } \\
\text { periodística }\end{array}$ & $\begin{array}{l}\text { Obtener un punto de } \\
\text { vista } \\
\text { Obtener información } \\
\text { sobre el contexto } \\
\text { Obtener información } \\
\text { como fuente }\end{array}$ & Escribir & $\begin{array}{l}\text { Noticia } \\
\text { Proyecto } \\
\text { Guión } \\
\text { Comunicado } \\
\text { Plan de trabajo } \\
\text { Artículo } \\
\text { científico } \\
\text { Nota en redes }\end{array}$ \\
\hline $\begin{array}{l}\text { Texto académico } \\
\text { Artículo } \\
\text { científico Norma } \\
\text { jurídica } \\
\text { Informe técnico }\end{array}$ & $\begin{array}{l}\text { Académica y } \\
\text { técnica }\end{array}$ & $\begin{array}{l}\text { Para la } \\
\text { comprensión y } \\
\text { crítica de géneros } \\
\text { de la esfera } \\
\text { profesional y } \\
\text { académica }\end{array}$ & $\begin{array}{l}\text { Usar referencia teórica } \\
\text { Ampliar o entender } \\
\text { un concepto Obtener } \\
\text { información general } \\
\text { sobre la temática }\end{array}$ & $\begin{array}{l}\text { Exponer } \\
\text { oralmente }\end{array}$ & $\begin{array}{l}\text { Exposición } \\
\text { oral } \\
\text { Entrevista }\end{array}$ \\
\hline Otros & Otras & $\begin{array}{l}\text { Para la } \\
\text { comprensión } \\
\text { general de textos }\end{array}$ & Diversos propósitos & $\begin{array}{l}\text { Producir texto } \\
\text { multimodal }\end{array}$ & $\begin{array}{l}\text { Pieza de } \\
\text { comunicación } \\
\text { visual }\end{array}$ \\
\hline
\end{tabular}

Fuente: Elaboración propia.

\section{Consideraciones Finales}

El objetivo de esta investigación, que tiene en esta encuesta apenas su primera fase, es reconocer las prácticas de referencia en lectura de géneros textuales en el campo profesional de la comunicación, sus sistemas de organización, así como las necesidades y desafíos que presentan para el actor experto.

La encuesta buscaba identificar cuáles eran las prácticas lectoras de los profesionales de la comunicación en Uruguay, y, mediante el análisis de los resultados, identificamos que estas incluyen leer para la producción de textos escritos y orales de la esfera profesional. Es decir, en esta comunidad, se lee para conseguir información y darle sentido a la misma. En esas prácticas de lectura, predominan la noticia como género de producción, así como las entrevistas y las exposiciones orales; aunque el elenco de posibles géneros producidos es muy extenso y variado. Aunque los participantes leen varios géneros para producir otros textos, algunos se destacan más: los de las esferas del periodismo (noticia, entrevista, artículo de opinión, editorial, reportaje) y los de las esferas del conocimiento técnico y académico (artículo científico, informe técnico, texto académico), además de otros como las normas jurídicas y las bases y convocatorias. Las finalidades principales de esas lecturas son la búsqueda de información sobre hechos, contextos y participantes, y la profundización sobre teorías, temáticas y puntos de vista, aspectos asociados con los contenidos más recurrentes de los géneros periodísticos, por un lado, y académicos y técnicos, por otro.

Las prácticas de literacidad disciplinar identificadas se asociación con estrategias de lectura que permiten abordar el contenido temático de los textos: sintetizar, contextualizar, contrastar y jerarquizar, así como analizar su material gráfico. También son comunes las estrategias y prácticas lectoras relacionadas con una orientación crítica al manejo de la información y las fuentes. Esos resultados nos llevan a suponer que algunas de esas operaciones estén más relacionadas con la lectura de géneros de la esfera periodística, y que otras sean para el procesamiento de textos de las esferas académica o técnica. Este aspecto es de vital importancia para profundizar la comprensión de la literacidad disciplinar en esta área, que es compleja debido 
a que los comunicadores leen géneros producidos en varias esferas de actividad diferentes, con finalidades diferentes, y movilizando para ello, estrategias diferentes. Aunque es necesario profundizar este punto en futuras etapas de nuestra investigación, resaltamos que, tal como constata Di Capua-Hidalgo (2016a) para la formación de grado en periodismo, los comunicadores desarrollan, para su ejercicio profesional, estrategias para la lectura de textos de la esfera académica o científica y que, por tanto, se debe valorar el papel de los géneros no periodísticos en la enseñanza universitaria del área de la comunicación.

Por último, el modelo de sistema de actividades trazado, además de incitarnos a profundizar la indagación de estas prácticas, nos ofrece algunas orientaciones para la planificación de los cursos de lectura respecto de:

- la importancia del trabajo integrado entre lectura y escritura, principalmente; y entre lectura y oralidad;

- la necesidad de jerarquizar el trabajo con géneros de las esferas periodística, académica y técnica, para el trabajo en lectura;

- la importancia de utilizar la escritura de los géneros noticia, entrevista y exposición oral como contexto praxiológico de la lectura;

- la pertinencia de proponer situaciones de aprendizaje en que los alumnos aborden textos de géneros de la esfera periodística para construir los escenarios de determinados hechos y que, además, esas situaciones les propongan la lectura de textos de géneros de las esferas académica y profesional para profundizar en la comprensión de las temáticas implicadas,

- la validez de profundizar el abordaje didáctico de las prácticas lectoras y las estrategias que sean funcionales para la comprensión de géneros de esferas diferentes, teniendo en cuenta el papel relevante ocupado por las estrategias de comprensión que implican una evaluación crítica de los textos.

Los hallazgos de esta primera parte de la investigación nos proveen insumos para construir una primera caracterización de las prácticas lectoras en el área de comunicación en Uruguay. Como limitaciones al estudio, debe mencionarse que no se utilizaron criterios de estadística descriptiva para ahondar en las tendencias cuantificadas y que aún no han sido discernidas las prácticas propias de cada área de ejercicio profesional específico, ni las diferencias entre las prácticas de profesionales novatos y con más experiencia. Asimismo, estudios posteriores requieren ahondar la comprensión de las nomenclaturas utilizadas por los profesionales para designar los diferentes géneros y las representaciones específicas relativas a qué serían esos géneros.

En futuras fases de esta investigación profundizaremos en la descripción de la literacidad disciplinar en el área de comunicación a través de entrevistas a informantes calificados y observaciones de prácticas de formación profesional. Estos datos permitirán luego potencialmente diseñar programas especializados en literacidad disciplinar a nivel terciario, como los propuestos en Navarro (2013), integrando la formación de docentes no especializados en la enseñanza de lenguas y estudiantes avanzados como tutores de pares. 


\section{REFERENCIAS}

ACHUGAR, M.; CARPENTER, B. Developing Disciplinary Literacy in a Multilingual History Classroom. Linguistics and Education, v. 23, n. 3, p. 262-276, 2012.

ACHUGAR, M.; STAINTON, C. Learning History and Learning Language: Focusing on Language in Historical Explanations to Support English Language Learners”. In: STEIN, M. K.; KUCAN, L. (org.). Instructional Explanations in the Disciplines. New York: Springer, 2010. p.145-169.

BAJTÍN, M. El problema de los géneros discursivos. In: BAJTÍN, M. Estética de la creación verbal. Buenos Aires: Siglo Veintiuno, 2008. p. 245-290.

BAZERMAN, C. Atos de Fala, Gêneros textuais e sistemas de atividades: como os textos organizam atividades e pessoas. In: DIONÍSIO, A.; HOFFNAGEL, J. (org.). Gêneros textuais, tipificação e interação. São Paulo: Cortez, 2005. p. 19-46.

BAZERMAN, C. La escritura de la organización social y la situación alfabetizada de la cognición: Extendiendo las implicaciones sociales de la escritura de Jack Goody. Revista Signos, v. 41, n. 68, p. 355380, 2008.

BERNSTEIN, B. Pedagogy, symbolic control and identity: theory, research, critique. Oxford: Rowman \& Littleford, 2000.

BRONCKART, J.-P.; DOLZ, J. A noção de competência: qual é sua pertinência para o estudo da aprendizagem das ações de linguagem? In: DOLZ, J.; OLLAGNIER, E. O enigma da competência em educação. Porto Alegre: Artmed, 2002, p. 29-46.

CALHOUN, C. Communication as Social Science (and more). International Journal of Communication, v. 5, p. 1479-1496, 2011.

DE PIETRO, J.-F.; SCHNEUWLY, B. O modelo do gênero: um conceito da engenharia didática. In: NASCIMENTO, E. (org.). Gêneros textuais. Da didática das línguas aos objetos de ensino. Campinas: Pontes, 2019. p. 51-81.

DI CAPUA-HIDALGO, S.. Alfabetização acadêmica do jornalismo em Chile: um estudo preliminar aos gêneros discursivos que se escrevem no processo de inserção disciplinar. Iniciação \& Formação Docente, v. 3, n. 2, p. 1-26, 2016a.

DI CAPUA-HIDALGO, S. La concepción de género desde el enfoque semiodiscursivo y la tradición periodística: una revisión teórica. Revista Información Pública, v. 7, n. 2, p. 295-318, 2016b.

DOLZ, J.; SCHNEUWLY, B. Gêneros e progressão em expressão oral e escrita - Elementos para reflexões sobre uma experiência suíça (francófona). In: SCHNEUWLY, B.; DOLZ, J. Gêneros orais e escritos na escola. São Paulo: Mercado de Letras, 2004. p. 35-60.

FISH, S. Is there a Text in this Class?: The Authority of Interpretive Communities. Cambridge, MA: Harvard University, 1980.

FRANCO, F. Entrando en el campo profesional. In: FRANCO, F.; KAPLÚN, G.; MARTÍNEZ, S. (org.). Vivir la universidad y la comunicación. Montevideo: Udelar, 2019. p. 53. 
HALLIDAY, M. A. K.; MARTIN, J. R. Writing Science: Literacy and Discursive Power. London: Falmer Press, 1993.

HYLAND, K. Disciplinary Discourses: Social Interactions in Academic Writing. London: Longman, 2004.

HYLAND, K. Genre and Academic Writing in the Disciplines. Language Teaching v. 41, n. 4, p. 543-562, 2008.

HYLAND, K. Genre, Discipline and Identity. Journal of English for Academic Purposes, v. 19, p. 32-43, 2015.

HYLAND, K. Specificity Revisited: How Far Should We Go Now? English for Specific Purposes, v. 21, p. 385-395, 2002.

JANSEN, H. The Logic of Qualitative Survey Research and its Position in the Field of Social Research Methods. Forum: Qualitative Social Research, v. 11, n. 2, p. 1-21, 2010.

LABELLA-SÁNCHEZ, N.; CRISTOVÃO, V. L. Gêneros de texto, capacidades de linguagem e Sistemas de atividades. In: RIESTRA, D.; TAPIA, S. M.; GOICOECHEA, M. V. (org.). Cuartas Jornadas Internacionales de investigación y prácticas en Didáctica de las lenguas y las literaturas. t. 2. Rio Negro: REUN, 2015. p. 513-493.

LEINHARDT, G.; STAINTON, C.; VIRJIJ, S. A Sense of History. Educational Psychologist, v. 29, n. 2, p. 79-88, 1994.

LEINHARDT, G.; YOUNG, K. M. Two Texts, Three Readers: Distance and Expertise in Reading History. Cognition and Instruction, v. 14, n. 4, p. 441-486, 1996.

MANGHI, D. Géneros en la enseñanza escolar: configuraciones de significado en clases de historia y biología desde una perspectiva multimodal. Revista Signos, v. 46, n. 82, p. 236-257, 2013.

MARCUSCHI, L. A. Da fala para escrita: atividades de retextualização. São Paulo: Cortez, 2007.

MARTIN, J. R.; ROSE, D. Genre Relations: Mapping Culture. London: Equinox, 2008.

MATON, K. Making Waves: a Key to Cumulative Knowledge-building. Linguistics \& Education, v. 24, p. 8-22, 2013.

MOJE, E. B. Foregrounding the Disciplines in Secondary Literacy Teaching and Learning: a Call for Change. Journal of Adolescent and Adult Literacy, v. 52, p. 96-107, 2008.

MOJE, E. Developing Socially Just Subject-matter Instruction: A Review of the Literature on Disciplinary Literacy Teaching. Review of Research in Education, v. 31, n. 1, p. 1-44, 2007.

MOSS, G. Textbook Language, Ideology and Citizenship. The Case of a History Textbook in Colombia. Functions of Language, v. 17, n. 1, p. 71-93, 2010.

MOYANO, E. Aportes del análisis de género y discurso a los procesos de enseñanza y aprendizaje escolares: las ciencias biológicas y la historia. Discurso \& Sociedad, v. 4, n. 2, p. 294-331, 2010.

NAVARRO, F. Trayectorias de formación en lectura y escritura disciplinar en carreras universitarias de humanidades. Diagnóstico y propuesta institucional. Revista Mexicana de Investigación Educativa, v. 18, n. 58, p. $709-734,2013$. 
NAVARRO, F.; AVILA REYES, N.; TAPIA-LADINO, M.; CRISTOVÃO, V.; MORITZ, M.; NARVÁEZ, E.; BAZERMAN, C. Panorama histórico y contrastivo de los estudios sobre lectura y escritura en educación superior publicados en América Latina. Revista Signos, v. 49, n. 1, p. 100-126, 2016.

PARODI, G. (org.). Alfabetización académica y profesional en el siglo XXI. Leer y escribir desde las disciplinas. Santiago de Chile: Ariel, 2010.

POTTER, J. The State of Media Literacy. Journal of Broadcasting \& Electronic Media, v. 54, n. 4, p. 675-696, 2010.

REISMAN, A. Reading Like a Historian: A Document-based History Curriculum Intervention in Urban High Schools. Cognition \& Instruction, v. 30, n. 1, p. 86-112, 2012.

RITCHIE, J.; LEWIS, J.; ELAM, G. Designing and Selecting Samples. In: MARUSTER, L; GILSENBERG, M. J. (org.). Qualitative Research Methods. London: Sage, 2003. p. 77-108.

SCHLEPPEGRELL, M. J. Supporting Disciplinary Learning Through Language Analysis: Developing Historical Literacy. In: CHRISTIE, F.; MATON, K. (org.). Disciplinarity: Functional Linguistic and Sociological Perspectives. London: Continuum, 2011. p. 197-216.

SCHLEPPEGRELL, M. The Language of Schooling: A Functional Linguistic Perspective. Mahwah, N.J:: Erlbaum, 2004.

SHANAHAN, T.; SHANAHAN, C. What is Disciplinary Literacy and Whys Does It Matter? Top Lang Disorders, v. 32, n. 1, p. 7-18, 2012.

SPIRES, H. A et al. Operationalizing and Validating Disciplinary Literacy in Secondary Education. Reading and Writing, v. 31, p. 1401-1434, 2018.

URUGUAY. Primera encuesta a egresados de ciencias de la comunicación de la Universidad de la República. In: SEMINARIO DEL OBSERVATORIO DE LAS PROFESIONES DE LA COMUNICACIÓN, 2., 20-21 jul. 2016, Montevideo. Anales [...]. Montevideo: Facultad de Información y Comunicación, 2016. Disponible en: https:/ / fic.edu.uy/InstComunicacion. Acceso en: 24 sept. 2020. p. 1-22.

WINEBURG, S. Historical Thinking and Other Unnatural Acts. Philadelphia: Temple University Press, 2001.

WINEBURG, S. Reading Abraham Lincoln: An Expert/expert Study in the Interpretation of Historical Texts. Cognitive Science, v. 22, n. 3, p. 319-346, 1998.

WINEBURG, S.; REISMAN, A. Disciplinary Literacy in History: A Toolkit for Digital Citizenship. Journal of Adolescent \& Adult Literacy, v. 58, n. 8, p. 636-639, 2015. 